Editorial

\title{
Metabolic pathway engineering
}

Organisms can be engineered to produce a wide variety of compounds by either enhancing endogenous metabolic pathways, or by introducing exogenous pathways that are either borrowed from other organism, or de novo-designed pathways unknown to nature. While overexpression of bottleneck enzymes and deletion of competing pathways remain at the core of metabolic pathway engineering, there are many other key elements that need to be considered to successfully develop strains for the production of valuable products. Some of these key elements include the choice of host organism, which must take into account the natural advantages and disadvantages of the host for a specific application; computational methods to discover, design, and optimize metabolic pathways; transcriptional control engineering, to finetune the levels and timing of enzyme expression; enzyme engineering to design novel enzymatic activities required in specific metabolic pathways; development of new synthetic biology tools to increase the capabilities and expedience of genetic interventions; and innovative strategies to reduce metabolic burden. This special issue of Synthetic and Systems Biotechnology, focusing on "Metabolic Pathway Engineering" comprises eight review articles and five original research studies that provide excellent examples of how these key elements support and augment metabolic engineering.

\section{Host organism choice}

Several articles raise the importance of selecting the appropriate host organism for the product and process of interest. Schwarz et al., shows that the actinomycete Amycolatopsis japonicum is a good host to produce the promising immunosuppressant brasilicardin A, where the producer strain Nocardia terpenica fell short [1]. The authors increase the production of this natural product in $A$. japonicum by overexpressing the mevalonate biosynthetic pathway to increasing intracellular levels of the IPP precursor, and expressing specific prenyltransferases that favor diterpenoid production. In another review article, Chen and Jian compare examples of strains of E. coli, Pseudomonas spp., and halophiles engineered for the production of polyhydroxyalkanoates [2], emphasizing the advantages of using extremophiles, such as halopholies, to avoid microbial contamination of industrial processes, and reduce the costs associated with process sterilization. The review by Löbs et al., give a current account of the challenges and opportunities of using nonconventional yeasts for metabolic engineering, with several examples of how these yeasts have already impacted industrial biotechnology [3]. Furthermore, the original study by Kildegaard et al., provides an excellent additional example of the vast potential of

Peer review under responsibility of KeAi Communications Co., Ltd. one such non-conventional yeast, Yarrowia lipolytica, to produce beta-carotenoids and other isoprenoids, specifically in their study for the production of astaxanthin [4].

\section{Computational methods and pathway control}

Since its beginnings, metabolic pathway engineering has greatly benefitted from computational models to study, design, and optimize metabolic pathways. In this special issue, Wang et al., review current tools for computational pathway design, focusing on the steps required to implement pathway design algorithms [5]. In their analysis, the authors classify these computational tools according to such algorithms into graph-based, stoichiometric-based, and retrosynthesis-based. Another review article, by Engstrom et al., give an update on the current state of transcriptional control engineering in bacteria, specifically on recent developments in cisfactors (promoters and terminators), trans-factors (transcription factors), and how these elements are combined to design transcriptional switches that respond to different signals [6]. They further discuss how some of these transcriptional control technologies have been applied to metabolic engineering and gene circuit designs. Furthermore, Barajas et al., contribute an excellent review on the current state of metabolic and enzyme engineering for the production of polyketides [7]. This review covers efforts to engineer polyketide synthase (PKS) activity both at the protein level and cellular level, emphasizing the importance of the synergy that must exist between the host, the availability of precursors in the context of metabolic engineering, and PKS protein engineering.

\section{Synthetic biology tools}

Two original studies describe the development of new synthetic biology tools for metabolic engineering. The article by Jin et al., describes a new toolbox that takes an evolutionary approach to construct prokaryotic regulatory element libraries with broad dynamic ranges in gene expression, by leveraging the mutation rate of synthetic DNA and the high efficiency of Gibson assembly [8]. The authors demonstrate this new technology to construct libraries of the T7 promoter library, lactose repressor binding site (lacO) library, prokaryotic ribosome binding site library, T7 terminator library and a trans-activating RNA library. These libraries may be useful for a wide range of applications, such as transcriptional and translational fine-tuning and sampling of the multidimensional gene expression space to balance multi-gene metabolic pathways. The second article on tool development, contributed by Domröse et al., describes a tool they call yTREX, which harnesses the versatility of yeast homologous recombination to carry out one-step cloning of vectors for the rapid TRansfer, chromosomal 
integration by random transposition, and EXpression of gene clusters in bacteria [9]. To demonstrate this tool, the authors assembled the multigene biosynthetic pathways for phenazine, violacein, and prodiginine (with as many as 14 genes), and integrated them into $P$. putida.

\section{Microbial consortia approaches}

Microbial consortia have been successfully used to spread out the metabolic burden of long and demanding heterologous pathways across multiple strains, improving titers and yields. In this special issue, Ganesan et al., report an excellent new example of how co-culture engineering can boost the production of natural products. In this case, the authors boost naringenin production by dividing the biosynthetic pathway between two different $E$. coli strains, which reduces the burden that each strain needs to endure [10]. Production of naringenin was further increased by optimizing by size of each inoculum, time of induction, and carbon source. Naringenin is an important precursor in the production of anthocyanins, a large family of plant natural products, made commonly in fruits and flowers as pigments, antioxidants, and antimicrobials, with various applications in the food and cosmetics industry. A review article by Zha et al., examines recent progress in metabolic engineering to produce anthocyanins. This review places special focus on enzyme screening, transporter engineering, cofactor overproduction, and process optimization for anthocyanin production in bacteria [11].

\section{New classes of molecules for metabolic pathway engineering}

Finally, this special issue contains two excellent reviews covering topics on new classes of products that have become accessible to metabolic engineering. One review, by Abdel-Mawgoud and Stephanopoulos, explores the potential to leverage the knowledge and technologies acquired from metabolic engineering efforts to produce lipids for biofuels, and apply them for the production of simple glycolipids, a class of compounds with a wide range of applications in the biomedical, pharmaceutical, and cosmetic industries [12]. The second review, contributed by Reed and Alper, delves into non-conventional metabolic pathways involving uncommon elements, such as halogens, selenium, metalloids, and metals. Such pathways may result in a number of new interesting applications, from the production of novel classes of chemical compounds via fermentation, to new applications in biomining and nanotechnology [13]. These reviews explore two of the most exciting frontiers in metabolic engineering.

We thank all contributing authors for making this special issue on "Metabolic Pathway Engineering" possible. We hope that readers find the articles it contains interesting and inspiring to their own research.

\section{References}

[1] Schwarz P, Roller L, Kulik A, Wohlleben W, Stegmann E. Engineering metabolic pathways in Amycolatopsis japonicum for the optimization of the precursor supply for heterologous brasilicardin congeners production. Synth Syst Biotechnol 2018.

[2] Chen G, Jiang X. Engineering bacteria for enhanced polyhydroxyalkanoates (PHA) biosynthesis. Synth Syst Biotechnol 2017;2(3):192-7.

[3] Löbs A, Schwartz C, Wheeldon I. Genome and metabolic engineering in nonconventional yeasts: current advances and applications. Synth Syst Biotechnol 2017;2(3):198-207.

[4] Kildegaard K, Adiego-Pérez B, Doménech Belda D, Khangura J, Holkenbrink C, Borodina I. Engineering of Yarrowia lipolytica for production of astaxanthin. Synth Syst Biotechnol 2017;2(4):287-94.

[5] Wang L, Dash S, Ng C, Maranas C. A review of computational tools for design and reconstruction of metabolic pathways. Synth Syst Biotechnol 2017;2(4): $243-52$.

[6] Engstrom M, Pfleger B. Transcription control engineering and applications in synthetic biology. Synth Syst Biotechnol 2017;2(3):176-91.

[7] Barajas J, Blake-Hedges J, Bailey C, Curran S, Keasling J. Engineered polyketides: synergy between protein and host level engineering. Synth Syst Biotechnol 2017;2(3):147-66.

[8] Jin E, Wong L, Jiao Y, Engel J, Holdridge B, Xu P. Rapid evolution of regulatory element libraries for tunable transcriptional and translational control of gene expression. Synth Syst Biotechnol 2017;2(4):295-301.

[9] Domröse A, Weihmann R, Thies S, Jaeger K, Drepper T, Loeschcke A. Rapid generation of recombinant Pseudomonas putida secondary metabolite producers using yTREX. Synth Syst Biotechnol 2017;2(4):310-9.

[10] Ganesan V, Li Z, Wang X, Zhang H. Heterologous biosynthesis of natural product naringenin by co-culture engineering. Synth Syst Biotechnol 2017;2(3): $236-42$.

[11] Zha J, Koffas M. Production of anthocyanins in metabolically engineered microorganisms: current status and perspectives. Synth Syst Biotechnol 2017;2(4):259-66.

[12] Abdel-Mawgoud A, Stephanopoulos G. Simple glycolipids of microbes: chemistry, biological activity and metabolic engineering. Synth Syst Biotechnol 2017.

[13] Reed K, Alper H. Expanding beyond canonical metabolism: interfacing alternative elements, synthetic biology, and metabolic engineering. Synth Syst Biotechnol 2017.

Hal S. Alper, Paul D. \& Betty Robertson Meek Centennial Professor, Frank A. Liddell, Jr. Centennial Fellow*

McKetta Department of Chemical Engineering, The University of Texas at Austin, USA

José L. Avalos, Assistant Professor and Pew Scholar Department of Chemical and Biological Engineering, Andlinger Center for Energy and the Environment, Princeton University, USA

* Corresponding author. E-mail address: halper@che.utexas.edu (H.S. Alper). 